\title{
PD-L1 associated with the expression of cancer cell-intrinsic PD-1 and p-S6 proteins and predicted a good prognosis in nasopharyngeal carcinoma
}

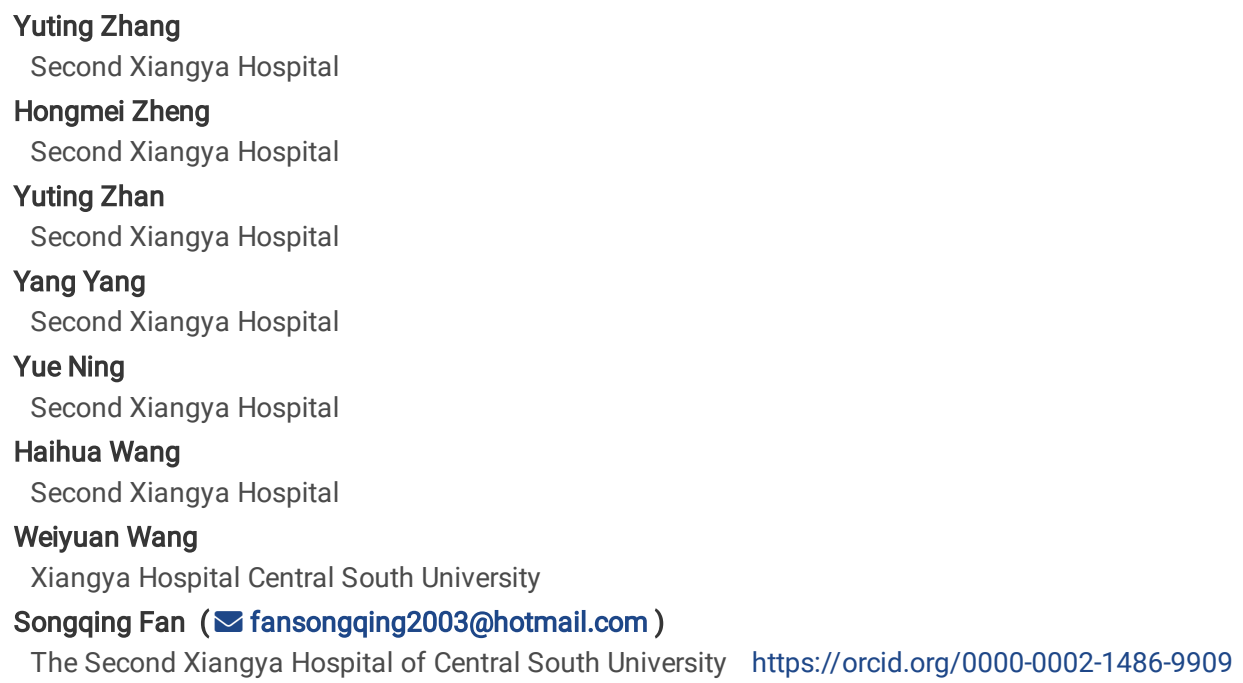




\begin{abstract}
Aims

programmed cell death ligand 1 (PD-L1) is the ligand of programmed death 1 (PD-1) which is a host immunity inhibitory receptor. PD-L1 expressed in tumor cells has being widely discussed, while there is little research about tumor intrinsic-PD-1. P-S6 is an important downstream effector of the PI3K/AKT/mTOR pathway. This study aimed to investigate the expression of PD-L1 protein and its relationship with cancer cell-intrinsic PD-1 protein and p-S6 in nasopharyngeal carcinoma (NPC).
\end{abstract}

\title{
Methods
}

the expression of PD-L1, PD-1 and p-S6 proteins in tissues of NPC, non-cancerous nasopharyngeal epithelia, primary lesions and matching metastases was detected by immunohistochemistry.

\section{Results}

PD-L1, PD-1 and p-S6 expression and co-expression of PD-L1 and PD-1 proteins were significantly higher in NPC than those of in the non-cancerous nasopharyngeal epithelia, respectively (all $P<0.05$ ). Furthermore, there was evidently elevated expression of PD-1 and co-expression of PD-L1 and PD-1 in matched metastasis of NPC compared to their primary lesions (all $P<0.01$ ). Overall survival rate for NPC patients with positive expression of PD-L1 ( $P=$ $0.035)$ was significantly higher than others. Multivariate Cox proportional hazard regression analysis identified that positive expression of PD-L $1(P=0.002)$ and p-S6 $(P=0.003)$ were independent prognostic factors for NPC patients.

\section{Conclusions}

positive expression of PD-L1 associated with expression of cancer cell-intrinsic PD-1 and p-S6, PD-L1 might be a good prognostic biomarker and p-S6 could serve as a valuable independent poor prognostic biomarker for NPC patients.

\section{Introduction}

Nasopharyngeal carcinoma (NPC) is one of the most common cancers in Asia, especially in southern China [1]. Epstein-Barr virus (EBV) is a recognized cause of NPC. Infection, genetic susceptibility, high nitrite food and smoking are also independent risk factors for NPC [2]. Nowadays, mainly treatments of NPC are radiotherapy and chemotherapy. Patients with early-stage of NPC could benefit from radiotherapy or/and chemotherapy, thereby obtaining a longer survival time $[3,4]$. Unfortunately, most NPC patients are already in the advanced stage at the time of diagnosis, and chemotherapy, radiotherapy, targeted therapy or immune treatment still cannot significantly effectively extend the survival time of them. [5-7]. In recent years, studies have found that immune escape of tumor cells and abnormal activation of signaling pathways play important roles in the occurrence and development of NPC [1]. Finding new targets of NPC will provide new clues for exploring more effective treatment of NPC patients.

Programmed death 1 (PD-1), a protein of the CD28 superfamily, is a cell membrane protein with 288 amino acids. The expression of tumor cell-intrinsic programmed death 1 (PD-1) played an important role in melanoma tumorigenesis [8]. As one of major ligands of PD-1, programmed death ligand 1 (PD-L1) also takes part in the tumor progression [9]. Ribosomal protein S6 (S6) could be activated by phosphorylated p70S6K which is a downstream effector of the AKT/mTOR pathway. Activated S6 is associated with poor prognosis of NPC via the messenger RNA translation machinery [10-11]. Recent research found that the cancer cell-intrinsic PD-1 activated by PD-L1 would promote phosphorylation of S6 and, initiate the translation process, which played an important role in the process of tumor occurrence, development, invasion and metastasis [12]. Besides, the cytoplasmic domain of PD-1 interacts with the S6 to promote phosphorylation of the mTOR effector protein, initiate the translation process of messenger RNA, and promote tumorigenesis, development, invasion and metastasis [9, 12-13]. But the role of PD-1, PD-L1 and S6 in NPC has not been studied yet.

In the present study, we detected expression of PD-L1, cancer cell-intrinsic PD-1 and p-S6 proteins in 281 cases of NPC and 51 cases of non-cancerous nasopharyngeal epithelia, as well as in 24 cases of primary NPC and their matched metastases, by immunohistochemistry (IHC), to investigate the relationship between the expression of PD-L1, cancer cell-intrinsic PD-1 or p-S6 proteins and clinicopathological features and prognostic implications in NPC.

\section{Materials And Methods}

\subsection{Ethics Statement}

All experimental protocols were approved by the Ethics Review Board of the Second Xiangya Hospital of Central South University (Scientific and Research Ethics Committee, No: Y202/2014), and all samples were obtained with informed consent. Also the written informed consent was obtained from the next of kin, caretaker, or guardians on the behalf of the minors/children participants who participated in our study.

\subsection{Tissue samples and clinical data}

All paraffin-embedded tissue samples, including 281 NPC tissues, 24 cases of primary NPC and their matched metastases, and 51 cases of non-cancerous control nasopharyngeal epithelia were collected from Department of pathology at the Second Xiangya Hospital of Central South University (Changsha, China) 
from January 2008 to December 2017. Complete clinical record, including follow-up data, was available for all patients. All cases were pathologically diagnosed and classified according to the latest WHO classification of head and neck tumors. These NPC patients did not receive radiotherapy or chemotherapy prior to biopsy. EB virus antibody and Epstein-Barr virus encoded RNAs (EBER) were detected by enzyme-linked immunosorbent assay (ELISA) and in-situ hybridization respectively. In present study cases, EBV antibody could be detected in peripheral blood of $99.6 \%$ cases of NPC patients and cases of 99.3\% NPC tissues were found to have the EBER-positive. Overall survival time was calculated from the date of diagnosis to the date of death or the last known date alive. All NPC were clinical staged according to the eighth edition of the UJCC/AJCC staging system. Characteristics of patient were presented in supplementary Table 1.

\section{$2.3 \mathrm{IHC}$ and scores}

IHC staining of PD-L1, PD-1 and p-S6 proteins was performed using ready-to-use MaxVisionTM+ HRP-Polymer anti-Mouse IHC Kit (Dako; Carpintrria, CA) on $4 \mu \mathrm{m}$ tissue sections. As described in our previous publication [11], the staining condition for each antibody was adjusted according to the laboratory experience. A 1:100 dilution of primary antibody to PD-L1 (Rabbit monoclonal antibody, Catalog \#ab228462, abcam, UK), PD-1 (Mouse polyclonal antibody,

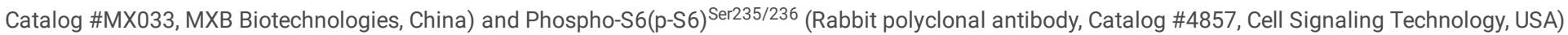
were used to detected the expression of those three proteins in the NPC and non-cancerous nasopharyngeal epithelia. Each experiment included positive and negative control slide. A matched IgG isotype antibody was used as a negative control to confirm the specificity of the antibody.

Immunohistochemical staining was independently evaluated under a light microscope at a magnification of $x 200$ by $Y$ Zhang and $Y$ Zhan blinded to the clinical data. The score was based on a semiquantitative method [14] as follows: cancer cell-intrinsic PD-1 was assessed as positive for NPC cells with a greater than 5 positive percentage. PD-L1 scored NPC by calculating combined positive scores (CPS). The CPS criterion is the ratio of the sum of positive tumor cells and positive infiltrating lymphocytes/macrophages relative to total tumor cells [11,13-14]. PD-L1 was regarded as positive when the score was higher than 5. A semiquantitative evaluation of p-S6 protein expression was performed using a method described in our previous paper [15]. Staining scores $\geq$ 2 was regarded as positive expression for an optimal cut-off value for $p$-S6. The two reviewers scored a repetition rate of $95 \%$, and the different parts were resolved by looking at paraffin sections and discussion again.

\subsection{Statistical analysis}

The correlation between expression of PD-L1, cancer cell-intrinsic PD-1, PD-L1, p-S6 and PD-L1/PD-1 proteins and clinicopathological features in NPC was analyzed by the chi-square test. The Spearman's rank correlation coefficient was used to assess the pairwise association between PD-L1, PD-1, p-S6 and PDL1/PD-1 expression in NPC. KaplanMeier analysis was hired for overall survival curves, and statistical significance was evaluated using the logrank test. Cox comparative hazards model was used to assess the independent prognostic factors of NPC with PD-L1, PD-1, p-S6 and PD-L1/PD-1 expression. All the above analysis was analyzed by SPSS (IBM SPSS Statistics 24.0) software. All P-values were based on two-sided statistical analysis, and when P<0.05, the data was considered to be statistically significant.

\section{Results}

3.1 Association between PD-L1, PD-1, p-S6 and combined PD-L1 and PD-1 expression and clinicopathological features of NPC

We detected the expression and subcellular localization of PD-L1, PD-1 and p-S6 proteins in NPC and non-cancerous control nasopharyngeal epithelia by IHC. Positive expression of PD-L1 protein was located in the membrane of NPC (Figure-1A). Positive expression of PD-1 protein was indicated in the cytoplasm of NPC (Figure-1B). Strong staining of p-S6 protein was discovered in the cytoplasm of NPC (Figure-1C) and the control normal nasopharyngeal epithelia (Figure1D). No staining of PD-L1 protein was found in the control normal nasopharyngeal epithelia (Figure-1E). Negative control was no staining in the NPC (Figure1F) (IHC, DAB staining, X200).

The positive expression of PD-L1, PD-1, p-S6 and co-expression of PD-1 and PD-L1 in NPC was 62.3\% (175/281), 22.1\% (62/281), 87.2\% (245/281), and 17.8\% (50/281), respectively. However, positive expression of PD-L1, PD-1, p-S6 and co-expression of PD-1 and PD-L1 in non-cancerous nasopharyngeal epithelia was 33.3\% (17/51), 7.8\% (4/51), 51.0\% (26/51) and 3.9(2/51), respectively. There was significantly higher positive expression of PD-L1, PD-1, p-S6 and coexpression of PD-1 and PD-L1 in NPC than those of in non-cancerous nasopharyngeal epithelia (Fig. 2A).

We further investigated PD-L1, PD-1, p-S6 and combined PD-1 and PD-L1 expression in the primary NPC and their matched lymph node metastases. Results in Fig. $2 \mathrm{~B}$ showed that the positive percentage of $\mathrm{PD}-1$ expression in metastasis $(62.5 \%, 15 / 24)$ was significantly higher than that in the matched primary lesions $(25.0 \%, 6 / 24)(P=0.009)$, as well as the co-expression of PD-1 and PD-L1 ( $P=0.009)$ (Fig. 2B). It's worth noting that in these primary and matched metastatic samples, all samples with PD-1 positive also acquired PD-L1 positive. However, there was no obvious difference in the expression of PD-L1 and p-S6 protein between primary NPC and their matched metastasis $(P>0.05)$.

We then investigated the relationship between PD-L1, PD-1, p-S6 or the co-expression of PD-L1/PD-1 proteins and clinicopathological features of NPC patients including gender, age, T/N/M classification, clinical stages, histological type and lymph node metastasis status. These results were shown in Table 1 . The positive percentage of PD-L1 ( $P=0.002)$ was significantly lower in patients with clinic T1 than those in T2, T3 and T4, but the patients with N0, N1 and N2 classifications was evidently higher than that N3 $(P=0.015)$. However, the positive percentage of PD-1 and co-expression of PD-L1 and PD-1, and p-S6 was not correlated with gender, $\mathrm{N}$ or $\mathrm{M}$ classification, clinical stage, histological type and lymph node status $(P>0.05$ for all). 
Table 1

Association between expression of PD-L1LPD-1 and p-S6 proteins and NPC clinicopathological features $(n=281)$

\begin{tabular}{|c|c|c|c|c|c|c|c|c|c|c|c|c|}
\hline \multirow{2}{*}{$\begin{array}{l}\text { Parameter(n) } \\
\text { Gender }\end{array}$} & \multicolumn{2}{|l|}{$\begin{array}{l}\text { PD-L1 } \\
\text { P (\%) N (\%) }\end{array}$} & \multirow[t]{2}{*}{$\begin{array}{l}P \text { - } \\
\text { values }\end{array}$} & \multicolumn{2}{|c|}{$\begin{array}{l}\text { PD-1 } \\
P(\%) N(\%)\end{array}$} & \multirow[t]{2}{*}{$\begin{array}{l}P \text { - } \\
\text { values }\end{array}$} & \multicolumn{2}{|l|}{$\begin{array}{l}\text { p-S6 } \\
P(\%) N(\%)\end{array}$} & \multirow[t]{2}{*}{$\begin{array}{l}P \text { - } \\
\text { values }\end{array}$} & \multicolumn{2}{|c|}{$\begin{array}{l}\text { PD-1/PD-L1\# } \\
\text { H (\%) L (\%) }\end{array}$} & \multirow[t]{2}{*}{$\begin{array}{l}P \text { - } \\
\text { values }\end{array}$} \\
\hline & & & & & & & & & & & & \\
\hline Male (203) & $130(64.0)$ & $73(36.0)$ & 0.326 & $46(22.7)$ & 157(77.3) & 0.697 & $181(89.2)$ & $22(10.8)$ & 0.110 & $38(18.7)$ & 165(81.3) & 0.513 \\
\hline Female (78) & $45(57.7)$ & $33(42.3)$ & & $16(20.5)$ & $62(79.5)$ & & 64(82.1) & $14(17.9)$ & & $12(15.4)$ & $66(84.6)$ & \\
\hline \multicolumn{13}{|l|}{ Age $(y r)^{\# \#}$} \\
\hline$\otimes 50(146)$ & $100(62.1)$ & 61(37.9) & 0.947 & $29(18.0)$ & $120(82.0)$ & 0.058 & $136(84.5)$ & $25(15.5)$ & 0.115 & $25(15.5)$ & $136(84.5)$ & 0.250 \\
\hline$\geq 50$ (135) & $75(62.5)$ & $45(37.5)$ & & $33(27.5)$ & $99(72.5)$ & & $109(90.8)$ & $11(9.2)$ & & $25(20.8)$ & $95(79.2)$ & \\
\hline \multicolumn{13}{|l|}{$\begin{array}{l}\text { Histological } \\
\text { type }\end{array}$} \\
\hline DNC (12) & $7(58.3)$ & $6(41.7)$ & 0.773 & $2(16.7)$ & 10(83.3) & 0.645 & $9(75.0)$ & $3(25.0)$ & 0.197 & $2(16.7)$ & 10(83.3) & 0.917 \\
\hline UDNC (269) & $168(62.5)$ & $101(37.5)$ & & $60(22.3)$ & 209(77.7) & & $236(87.7)$ & $33(12.3)$ & & $48(17.8)$ & $221(82.2)$ & \\
\hline \multicolumn{13}{|l|}{$\begin{array}{l}\text { T- } \\
\text { classification }\end{array}$} \\
\hline T1 (25) & $7(28.0)$ & 18(72.0) & $0.002^{\star \star}$ & $5(20.0)$ & $20(80.0)$ & 0.921 & 19(76.0) & $6(24.0)$ & 0.357 & $2(8.0)$ & $23(92.0)$ & 0.317 \\
\hline T2 (103) & $65(63.1)$ & $38(36.9)$ & & $23(22.3)$ & $80(77.7)$ & & 91(88.3) & 12(11.7) & & $16(15.5)$ & $87(84.5)$ & \\
\hline T3 (78) & $51(65.4)$ & $27(34.6)$ & & $19(24.4)$ & $59(75.6)$ & & 68(87.2) & $10(12.8)$ & & 18(23.1) & $60(76.9)$ & \\
\hline T4 (75) & $52(69.3)$ & 23(30.7) & & $15(20.0)$ & $60(80.0)$ & & 67(89.3) & $8(10.7)$ & & 14(18.7) & 61(81.3) & \\
\hline \multicolumn{13}{|l|}{$\begin{array}{l}\mathrm{N}- \\
\text { classification }\end{array}$} \\
\hline N0 (46) & $29(63.0)$ & 17(37.0) & $0.015^{\star}$ & 14(30.4) & $32(60.6)$ & 0.416 & 43(93.5) & $3(6.5)$ & 0.180 & $10(21.7)$ & $36(78.3)$ & 0.729 \\
\hline N1 (82) & 49(59.8) & $33(40.2)$ & & 16(19.5) & $66(80.5)$ & & 72(87.8) & $10(12.2)$ & & 13(15.9) & $69(84.1)$ & \\
\hline N2 (122) & $85(69.7)$ & $37(30.3)$ & & 24(19.7) & $98(80.3)$ & & $101(82.8)$ & $21(17.2)$ & & 23(18.9) & $99(81.1)$ & \\
\hline N3 (31) & 12(38.7) & 19(61.3) & & $8(25.8)$ & $23(74.2)$ & & $29(93.5)$ & $2(6.5)$ & & $4(12.9)$ & $27(87.1)$ & \\
\hline \multicolumn{13}{|l|}{$\begin{array}{l}\text { M- } \\
\text { classification }\end{array}$} \\
\hline M0 (272) & $171(62.9)$ & 101(37.1) & 0.262 & $59(21.7)$ & 213(78.3) & 0.407 & $237(87.1)$ & $35(12.9)$ & 0.887 & $48(17.6)$ & $224(82.4)$ & 0.7249 \\
\hline M1 (9) & $4(44.4)$ & $5(55.6)$ & & $3(33.3)$ & $6(66.7)$ & & $8(88.9)$ & 1(11.1) & & $2(22.2)$ & $7(77.8)$ & \\
\hline \multicolumn{13}{|l|}{$\begin{array}{l}\text { Clinical } \\
\text { stage }\end{array}$} \\
\hline $\begin{array}{l}\bigotimes \text { and } \otimes(n= \\
57)\end{array}$ & $34(59.6)$ & $23(40.4)$ & 0.647 & 10(17.5) & $47(82.5)$ & 0.357 & $52(91.2)$ & $5(8.8)$ & 0.307 & $7(12.3)$ & $50(87.7)$ & 0.223 \\
\hline$\nabla$ and $\nabla(224)$ & $141(62.9)$ & 83(37.1) & & $52(23.2)$ & 172(76.8) & & 193(86.2) & $31(13.8)$ & & 43(19.2) & 181(80.8) & \\
\hline \multicolumn{13}{|l|}{$\begin{array}{l}\text { Lymph node } \\
\text { status }\end{array}$} \\
\hline LNM (235) & $146(62.1)$ & $89(37.9)$ & 0.907 & $48(20.4)$ & 187(79.6) & 0.134 & $202(86.0)$ & $33(14.0)$ & 0.163 & $40(17.0)$ & 195(83.0) & 0.444 \\
\hline No LNM (46) & $29(63.0)$ & 17(37.0) & & 14(30.4) & $32(69.6)$ & & 43(93.5) & $3(6.5)$ & & $10(21.7)$ & $36(78.3)$ & \\
\hline
\end{tabular}

Abbreviations: NPC nasopharyngeal carcinoma; DNPC differentiated non-keratinizing nasopharyngeal carcinoma; UDNPC undifferentiated non-keratinizing nasopharyngeal carcinoma; LNM lymph node metastasis; $N$ negative; $P$ positive. ${ }^{\#}$ Co-expression of PD- 1 and PD-L1; \#\# the average age of all subjects was 49.8 years; ${ }^{*}$ Correlation is significant at the $p<0.05$ level (two tailed). ${ }^{\star *}$ Correlation is significant at the $p<0.01$ level (two tailed).

\subsection{Correlations of PD-L1, PD-1, p-S6 and co-expression of PD-L1 and PD-1 proteins expression in NPC}

There was a notable phenomenon that in primary and matched metastatic NPC tissues, samples with positive PD-1 expression were accompanied by positive PD-L1, which attracted our attentions (Fig. 2B). Therefore, we investigated whether there were some correlations among these proteins. The association between PD-L1, PD-1, p-S6 and co-expression of PD-L1/PD-1 proteins in 281 NPC cases was shown in Table 2. Expression of PD-L1 was positively associated with PD-1 $(r=0.219, P<0.001)$, p-S6 $(r=0.273, P<0.001)$ or co-expression of PD-L1 and PD-1 $(r=0.366, P<0.001)$ in NPC. In addition, $p-S 6$ was also positively 
related to PD-1 ( $r=0.127, P=0.033)$ and co-expression of PD-L1 and PD-1 in NPC $(r=0.153, P=0.01)$. Consistent with the notable phenomenon, PD-1 expression was strongly related with combined PD-L1 and PD-1 expression $(r=0.885, P<0.001)$.

Table 2

The pairwise correlation between expression of PD-L1, PD-1 and p-S6 proteins in the 281 cases of NPC

\begin{tabular}{|c|c|c|c|c|}
\hline & PD-L1 & PD-1 & p-S6 & PD-1/PD-L1 \# \\
\hline \multicolumn{5}{|l|}{ PD-L1 } \\
\hline Values & - & 0.219 & 0.273 & 0.366 \\
\hline Significant & & $0.000 * \star$ & $0.000 * *$ & $0.000 * *$ \\
\hline \multicolumn{5}{|l|}{ PD-1 } \\
\hline Values & - & - & 0.127 & 0.885 \\
\hline Significant & & & $0.033^{*}$ & $0.000 * *$ \\
\hline \multicolumn{5}{|l|}{ p-S6 } \\
\hline Values & - & - & - & 0.153 \\
\hline \multicolumn{3}{|l|}{ Significant } & & $0.010^{*}$ \\
\hline
\end{tabular}

3.3 The impact of PD-L1, PD-1, p-S6 and combined PD-1and PD-L1 proteins expression on prognosis of NPC patients.

Kaplan-Meier survival curves using log-rank test were applied to analyze survival status of NPC patients with differentially expressed PD-L1, PD-1, p-S6 and combined PD-L1 and PD-1 proteins. The Fig. 3 showed the Kaplan-Meier survival curves for NPC patients with the different variables described above. Overall survival rate for NPC patients with positive expression of PD-L1 $(P=0.035$, Fig. 3A) was significantly higher than that for patients with negative PD-L1 expression. On the contrary, NPC patients with positive expression of PD-1 $(P=0.031$, Fig. 3B) p-S6 $(P=0.044$, Fig. $3 C)$ or combined PD-1 and PD-L1 ( $P=0.042$, Fig. 3D) had shorter survival time than others by univariate analysis.

Furthermore, we investigated whether PD-L1, PD-1, p-S6 and combined PD-L1 and PD-1 proteins expression could be used as independent prognostic factors for NPC patients using multivariate Cox proportional hazard regression analysis. The results in Table 3 showed that positive expression of PD-L1 protein was identified as an independent good prognostic factor for overall survival of NPC patients $(P=0.002)$, but positive expression of p-S6 protein was identified as an independent poor prognostic factor for overall survival of NPC patients $(P=0.003)$, as well as LNM status $(P=0.004), \mathrm{N}$-classification $(P<0.001)$, Mclassification $(P<0.001)$ and clinical stage $(P=0.005)$. However, patients with positive expression of PD-1 and co-expression of PD-L1 and PD-1 had no significant impact on the overall survival status of NPC patients $(P>0.05$, respectively). Other factors including gender, age, histological type and T/N classification also have no obvious impacts on the prognosis of NPC patients (all $P>0.05$ ). 
Table 3

Summary of multivariate Cox proportional hazard regression analysis used to evaluate overall survival in 281 cases of NPC patients.

\begin{tabular}{|c|c|c|c|c|c|c|}
\hline \multirow{3}{*}{$\begin{array}{l}\text { Parameter } \\
\text { Gender }\end{array}$} & \multirow{3}{*}{$\begin{array}{l}\text { SE } \\
0.305\end{array}$} & \multirow{3}{*}{$\begin{array}{l}\text { Wald } \\
0.626\end{array}$} & \multirow{3}{*}{$\begin{array}{l}\text { Significance } \\
0.429\end{array}$} & \multirow{3}{*}{$\begin{array}{l}\operatorname{Exp}(B) \\
0.785\end{array}$} & \multirow{2}{*}{\multicolumn{2}{|c|}{$\begin{array}{l}\text { 95.0\% Cl for } \operatorname{Exp}(\mathrm{B}) \\
\text { Lower Upper }\end{array}$}} \\
\hline & & & & & & \\
\hline & & & & & 0.432 & 1.429 \\
\hline Age & 0.251 & 2.736 & 0.098 & 1.516 & 0.926 & 2.481 \\
\hline Histological type & 1.017 & 0.759 & 0.384 & 0.412 & 0.056 & 3.024 \\
\hline LNM status & 0.412 & 8.504 & $0.004^{\star \star}$ & 3.329 & 1.483 & 7.470 \\
\hline T-classification & 0.169 & 1.090 & 0.297 & 1.193 & 0.856 & 1.662 \\
\hline N-classification & 0.167 & 12.792 & $0.000 * *$ & 1.817 & 1.310 & 2.521 \\
\hline M-classification & 0.452 & 16.961 & $0.000 * *$ & 6.426 & 2.651 & 15.575 \\
\hline Clinical stages & 0.176 & 7.830 & $0.005^{\star \star}$ & 1.637 & 1.159 & 2.312 \\
\hline PD-L1 & 0.301 & 9.434 & $0.002^{\star \star}$ & 0.397 & 0.220 & 0.716 \\
\hline PD-1 & 0.501 & 1.626 & 0.202 & 1.895 & 0.709 & 5.064 \\
\hline p-S6 & 0.481 & 8.665 & $0.003^{\star *}$ & 4.115 & 1.604 & 10.556 \\
\hline PD-L1/PD-1 & 0.607 & 0.170 & 0.680 & 1.284 & 0.391 & 4.217 \\
\hline
\end{tabular}

Abbreviations: SE, standard error (SE); Exp (B), exponentiation of the B coefficient; Cl, confidence interval.; LNM lymph node metastasis; NPC nasopharyngeal carcinoma.

Note: multivariate analysis of Cox proportional hazard regression, ${ }^{*}$ Correlation is significant at the $p<0.05$ level (two tailed). ${ }^{*}$ Correlation is significant at the $p<0.01$ level (two tailed).

\section{Discussion}

PD-L1, as a ligand for PD-1, is a transmembrane protein encoded by the $C d 274$ gene expressing on immune cells and tumor cells. It is not only a cancerpromoting factor in some certain malignant tumors such as hepatocellular carcinoma, gastric cancer and esophageal cancer; but also a protective factor in some other tumors including merkel cell carcinoma and breast cancer [16-20]. However, the mechanism of PD-L1 action in NPC is not clear [21, 22]. Previous studies indicated that short overall survival time of NPC patients was associated with high expression of PD-L1 [23, 24]. But other research results showed that patients with high expression of PD-L1 had a better prognosis [25-27], which was in line with our results. We found that there was significant higher positive expression of PD-L1, PD-1, p-S6 and combined PD-1 and PD-L1 in NPC than that of in non-cancerous nasopharyngeal epithelia. In addition, PD-L1 expression had effect on T-classification of NPC. In conclusion, overexpression of PD-L1, PD-1, combined PD-L1 and PD-1 and p-S6 were all related with tumorigenesis.

Cox multivariate regression analysis showed that PD-L1 positive expression in NPC patients had a lower risk of death. In fact, the relationship between PD-L1 expression and tumor immune evasion is not directly proportional, and may just represent the persistence of anti-tumor response [28]. Here's more evidence that PD-L1 could take part in the regulation of anti-tumor immunity. It was found that AhR (Aryl hydrocarbon receptor) activation could induce PD-1 expression in CD8 + T. Maybe their co-stimulators AhR induced the correlation expression between PD-1 and combined PD-L1 and PD-1 expression [29, 30]. The secretion of interferon gamma (IFNY) and the up-regulation of tumor PD-L1 expression promote each other, resulting in increased infiltration of $\mathrm{CD}^{+}{ }^{+}$and $\mathrm{CD} 3^{+} \mathrm{T}$ cells around NPC tissues, and the accumulation of these immune cells promotes the development of anti-tumor immunity [31-33].

PD-L1 and PD-L2, as ligands of PD-1, are mainly expressed in antigen presenting cells and tumor cells. And they will inhibit the tumor killing activity of T cells and down-regulate the $T$ cell response when they bind to PD-1. The transmembrane receptor PD-1 is a prominent checkpoint receptor primarily expressed on $T$ cells [34, 35]. PD-1 plays a vital role in promoting NPC growth and is associated with the short overall survival time in patients with NPC [36]. Previous studies about the expression of PD-1 in NPC have primarily examined the expression of PD-1 in lymphocytes [37-38]. It was firstly reported that the expression of PD1 was also presented in cancer cells and promoted the occurrence and development of tumors in melanoma [8]. Other studies have also shown that the expression of cell-intrinsic PD-1 promotes the development of liver cancer and pancreatic cancer, and the survival rate of patients with high expression is lower $[39,40]$. Tumor cell-intrinsic PD-1 promotes the occurrence of melanoma and hepatocellular carcinoma by activating the mTOR signaling [12]. In pancreatic cancer, cell-intrinsic PD-1 promoted tumor growth through the Hippo signaling pathway outside the immune system [40]. However, Du et al found that cellintrinsic PD-1 was presented in NSCLC as a tumor suppressor [41]. In our study, we found that patients with positive PD-1 expression in NPC tissues had shorter survival time, which is consistent with findings in most malignancies. We also found that the positive expression rate of cancer cell-intrinsic PD-1 in metastatic NPC lesions was obviously higher than that in primary lesions. Although there was no significant difference in PD-1 expression in primary NPC with or without lymph node metastasis, it was worth noting that the immune morphology of NPC cells altered during distant metastasis. Consequently, we speculated that cell-intrinsic PD-1 might participate in the distant metastases of NPC. However, the number of matched primary/metastatic and lymph node metastases is limited, so further experimentation with larger size is needed to confirm the relationship between expression of PD-1 and the NPC metastasis.

The PI3K signaling pathway is involved in the regulation of PD-L1 expression. And S6 is a target downstream of the PI3K/AKT/mTOR signaling pathway. Overexpression of p-S6 led to dysregulation of the mTOR signaling pathway [42-44]. The role of PD-1 in the promotion of S6 phosphorylation resulted in tumor proliferation in melanoma [12]. Our results showed that patients with positive p-S6 expression had shorter overall survival, and there was a correlation 
between p-S6 expression and PD-1 and PD-L1 expression. At the same time, the p-S6 positive rate in patients with co-expression of PD-1 and PD-L1 was significantly higher than other patients. Hence, we speculated that PD-1 may also play an encouraging role in S6 phosphorylation in NPC. In conclusion, expression of PD-L1, PD-1, combined PD-L1 and PD-1 and p-S6 are correlational, especially between PD-1 and combined PD-L1 and PD-1 expression. But, these need to be further verified by cell lines and animal models in the following experiments.

In summary, in our study, there was significantly higher positive expression of PD-L1, PD-1, p-S6 and combined PD-1 and PD-L1 proteins in NPC. Furthermore, PD-1 might also be involved in the metastatic spread of NPC. Positive expression of PD-L1 associated with expression of PD-1 and p-S6, Positive expression of PD-L1 and p-S6 could serve as valuable independent prognostic biomarkers for NPC patients.

\section{Declarations}

\section{Ethics approval and consent to participate}

The study was obtained with informed consent.

\section{Consent for publication}

The consent for publication was obtained of the study.

\section{Availability of data and material}

All the data and material were available.

\section{Competing interests}

The authors have no conflicts of interest to declare.

\section{Funding}

The work was supported by the National Natural Sciences Foundations of China (No: 81972838, 81472773, 81773218, 81703009, 81802791 and 81272566) and The Natural Sciences Foundations of Hunan Province (No: 2018JJ3858 and 2017JJ3457).

Authors' contributionsSongqing Fan designed and revised the manuscript. Yuting Zhang wrote the manuscript and made the tables. Hongmei Zheng and Yuting Zhan collected clinical statistics. Yang Yang, Yue Ning and Haihua Wang participated in the experiment. Weiyuan Wang checked the manuscript and gave the suggestions.

\section{Acknowledgements}

The work was supported by the National Natural Sciences Foundations of China (No: 81972838, 81472773, 81773218, 81703009, 81802791 and 81272566 ) and The Natural Sciences Foundations of Hunan Province (No: 2018JJ3858 and 2017JJ3457).

\section{References}

1. Cho WC. Nasopharyngeal carcinoma: molecular biomarker discovery and progress. MOL CANCER. 2007;6:1.

2. Chen YP, Chan A, Le QT, Blanchard P, Sun Y, Ma J. Nasopharyngeal carcinoma. LANCET. 2019;394:64-80.

3. Tuan JK, Ha TC, Ong WS, Siow TR, Tham IW, Yap SP, et al. Late toxicities after conventional radiation therapy alone for nasopharyngeal carcinoma. RADIOTHER ONCOL. 2012;104:305-11.

4. Qu Y, Chen Y, Yu H, Zhao Y, Chen G, Bai L, et al. Survival and Prognostic Analysis of Primary Nasopharyngeal Carcinoma in North China. CLIN LAB. 2015;61:699-708.

5. Wu F, Wang R, Lu H, Wei B, Feng G, Li G, et al. Concurrent chemoradiotherapy in locoregionally advanced nasopharyngeal carcinoma: treatment outcomes of a prospective, multicentric clinical study. RADIOTHER ONCOL. 2014;112:106-11.

6. Yang H, Chen X, Lin S, Rong J, Yang M, Wen Q, et al. Treatment outcomes after reduction of the target volume of intensity-modulated radiotherapy following induction chemotherapy in patients with locoregionally advanced nasopharyngeal carcinoma: A prospective, multi-center, randomized clinical trial. RADIOTHER ONCOL. 2018;126:37-42.

7. Xiao Z, Chen Z. Deciphering nasopharyngeal carcinoma pathogenesis via proteomics. Expert Rev Proteomics. 2019;16:475-85.

8. Kleffel S, Posch C, Barthel SR, Mueller H, Schlapbach C, Guenova E, et al. Melanoma Cell-Intrinsic PD-1 Receptor Functions Promote Tumor Growth. CELL. 2015;162:1242-56.

9. Ishida Y, Agata Y, Shibahara K, Honjo T. Induced expression of PD-1, a novel member of the immunoglobulin gene superfamily, upon programmed cell death. EMBO J. 1992;11:3887-95.

10. Chung J, Kuo CJ, Crabtree GR, Blenis J. Rapamycin-FKBP specifically blocks growth-dependent activation of and signaling by the 70 kd S6 protein kinases. CELL. 1992;69:1227-36.

11. Wang W, Wen Q, Xu L, Xie G, Li J, Luo J, et al. Activation of Akt/mTOR pathway is associated with poor prognosis of nasopharyngeal carcinoma. PLOS ONE. 2014;9:e106098. 
12. Yao H, Wang H, Li C, Fang JY, Xu J. Cancer Cell-Intrinsic PD-1 and Implications in Combinatorial Immunotherapy. FRONT IMMUNOL. 2018;9:1774.

13. de Vicente JC, Pena I, Rodrigo JP, Rodriguez-Santamarta T, Lequerica-Fernandez P, Suarez-Fernandez L, et al. Phosphorylated ribosomal protein S6 correlation with p21 expression and inverse association with tumor size in oral squamous cell carcinoma. Head Neck. 2017;39:1876-87.

14. Bellmunt J, de Wit R, Vaughn DJ, Fradet Y, Lee JL, Fong L, et al. Pembrolizumab as Second-Line Therapy for Advanced Urothelial Carcinoma. N Engl J Med. 2017;376:1015-26.

15. Feng J, Xie G, Zhan Y, Lu J, Xu L, Fan S, Wang W. Elevated HSP90 associates with expression of HIF-1a and p-AKT and is predictive of poor prognosis in nasopharyngeal carcinoma. Histopathology. 2019;75(2):202-12.

16. D'Alterio C, Nasti G, Polimeno M, Ottaiano A, Conson M, Circelli L, et al. CXCR4-CXCL12-CXCR7, TLR2-TLR4, and PD-1/PD-L1 in colorectal cancer liver metastases from neoadjuvant-treated patients. Oncoimmunology. 2016;5(12):e1254313.

17. Boger C, Behrens HM, Mathiak M, Kruger S, Kalthoff H, Rocken C. PD-L1 is an independent prognostic predictor in gastric cancer of Western patients. Oncotarget. 2016;7(17):24269-83.

18. Yagi T, Baba Y, Ishimoto T, Iwatsuki M, Miyamoto Y, Yoshida N, et al. PD-L1 Expression, Tumor-infiltrating Lymphocytes, and Clinical Outcome in Patients With Surgically Resected Esophageal Cancer. Ann Surg. 2019;269(3):471-8.

19. Giraldo NA, Nguyen P, Engle EL, Kaunitz GJ, Cottrell TR, Berry S, et al. Multidimensional, quantitative assessment of PD-1/PD-L1 expression in patients with Merkel cell carcinoma and association with response to pembrolizumab. J Immunother Cancer. 2018;6(1):99.20.

20. Tsang JY, Au WL, Lo KY, Ni YB, Hlaing T, Hu J, et al. PD-L1 expression and tumor infiltrating PD-1 + lymphocytes associated with outcome in HER2 + breast cancer patients. Breast Cancer Res Treat. 2017;162(1):19-30.

21. Keir ME, Butte MJ, Freeman GJ, Sharpe AH. PD-1 and its ligands in tolerance and immunity. ANNU REV IMMUNOL. 2008;26:677-704.

22. Wang X, Teng F, Kong L, Yu J. PD-L1 expression in human cancers and its association with clinical outcomes. Onco Targets Ther. 2016;9:5023-39.

23. Zhou Y, Shi D, Miao J, Wu H, Chen J, Zhou X, et al. PD-L1 predicts poor prognosis for nasopharyngeal carcinoma irrespective of PD-1 and EBV-DNA load. Sci Rep. 2017;7:43627.

24. Zheng L, Cao C, Cheng G, Hu Q, Chen X. Cytomembranic PD-L1 expression in locoregionally advanced nasopharyngeal carcinoma. Onco Targets Ther. 2017;10:5483-7.

25. Zhu Q, Cai MY, Chen CL, Hu H, Lin HX, Li M, et al. Tumor cells PD-L1 expression as a favorable prognosis factor in nasopharyngeal carcinoma patients with pre-existing intratumor-infiltrating lymphocytes. ONCOIMMUNOLOGY. 2017;6:e1312240.

26. Lee VH, Lo AW, Leung CY, Shek WH, Kwong DL, Lam KO, et al. Correlation of PD-L1 Expression of Tumor Cells with Survival Outcomes after Radical Intensity-Modulated Radiation Therapy for Non-Metastatic Nasopharyngeal Carcinoma. PLOS ONE. 2016;11:e157969.

27. Liu YJ, Tsang NM, Hsueh C, Yeh CJ, Ueng SH, Wang TH, et al. Low PD-L1 Expression Strongly Correlates with Local Recurrence in Epstein-Barr VirusPositive Nasopharyngeal Carcinoma after Radiation-Based Therapy. Cancers (Basel) (2018) 10.

28. Taube JM, Anders RA, Young GD, Xu H, Sharma R, McMiller TL, et al. Colocalization of inflammatory response with B7-h1 expression in human melanocytic lesions supports an adaptive resistance mechanism of immune escape. SCI TRANSL MED. 2012;4:127r-137r.

29. Liu Y, Liang X, Dong W, Fang Y, Lv J, Zhang T, et al. Tumor-Repopulating Cells Induce PD-1 Expression in CD8(+) T Cells by Transferring Kynurenine and AhR Activation. Cancer Cell. 2018;33(3):480-94. e7.

30. Wang GZ, Zhang L, Zhao XC, Gao SH, Qu LW, Yu H, et al. The Aryl hydrocarbon receptor mediates tobacco-induced PD-L1 expression and is associated with response to immunotherapy. Nat Commun. 2019;10(1):1125.

31. Taube JM, Anders RA, Young GD, Xu H, Sharma R, McMiller TL, et al. Colocalization of inflammatory response with B7-h1 expression in human melanocytic lesions supports an adaptive resistance mechanism of immune escape. SCI TRANSL MED. 2012;4:127r-137r.

32. Spranger S, Spaapen RM, Zha Y, Williams J, Meng Y, Ha TT, et al Up-regulation of PD-L1, IDO, and T(regs) in the melanoma tumor microenvironment is driven by CD8(+) T cells. SCI TRANSL MED(2013) 5: 116r-200r.

33. Ohigashi $Y$, Sho M, Yamada $Y$, Tsurui $Y$, Hamada $K$, Ikeda N, et al. Clinical significance of programmed death-1 ligand-1 and programmed death-1 ligand-2 expression in human esophageal cancer. CLIN CANCER RES. 2005;11:2947-53.

34. Topalian SL, Drake CG, Pardoll DM. Targeting the PD-1/B7-H1(PD-L1) pathway to activate anti-tumor immunity. CURR OPIN IMMUNOL. 2012;24:207-12.

35. Wang Y, Wang H, Yao H, Li C, Fang JY, Xu J. Regulation of PD-L1: Emerging Routes for Targeting Tumor Immune Evasion. FRONT PHARMACOL. 2018;9:536.

36. Jiang F, Yu W, Zeng F, Cheng G, Xu J, Yang S, et al. PD-1 high expression predicts lower local disease control in stage IV M0 nasopharyngeal carcinoma. BMC Cancer. 2019;19(1):503.

37. Tang Y, He Y, Shi L, Yang L, Wang J, Lian Y, et al. Co-expression of AFAP1-AS1 and PD-1 predicts poor prognosis in nasopharyngeal carcinoma. Oncotarget. 2017;8:39001-11.

38. Hsu MC, Hsiao JR, Chang KC, Wu YH, Su IJ, Jin YT, et al. Increase of programmed death-1-expressing intratumoral CD8 T cells predicts a poor prognosis for nasopharyngeal carcinoma. Mod Pathol. 2010;23:1393-403.39.

39. Li H, Li X, Liu S, Guo L, Zhang B, Zhang J, et al Programmed cell death-1 (PD-1) checkpoint blockade in combination with a mammalian target of rapamycin inhibitor restrains hepatocellular carcinoma growth induced by hepatoma cell-intrinsic PD-1. HEPATOLOGY (2017) 66: 1920-1933.

40. Pu N, Gao S, Yin H, Li JA, Wu W, Fang Y, et al. Cell-intrinsic PD-1 promotes proliferation in pancreatic cancer by targeting CYR61/CTGF via the hippo pathway. CANCER LETT. 2019;460:42-53. 
41. Du S, McCall N, Park K, Guan Q, Fontina P, Ertel A, et al. Blockade of Tumor-Expressed PD-1 promotes lung cancer growth. ONCOIMMUNOLOGY. 2018;7:e1408747.

42. Chen J, Jiang CC, Jin L, Zhang XD. Regulation of PD-L1: a novel role of pro-survival signaling in cancer. ANN ONCOL. 2016;27:409-16.

43. Yang C, Peng J, Jiang W, Zhang Y, Chen X, Wu X, et al. mTOR activation in immature cells of primary nasopharyngeal carcinoma and anti-tumor effect of rapamycin in vitro and in vivo. CANCER LETT. 2013;341:186-94.

44. Huang XM, Dai CB, Mou ZL, Wang LJ, Wen WP, Lin SG, et al. Overproduction of cyclin D1 is dependent on activated mTORC1 signal in nasopharyngeal carcinoma: implication for therapy. CANCER LETT. 2009;279:47-56.

\section{Figures}
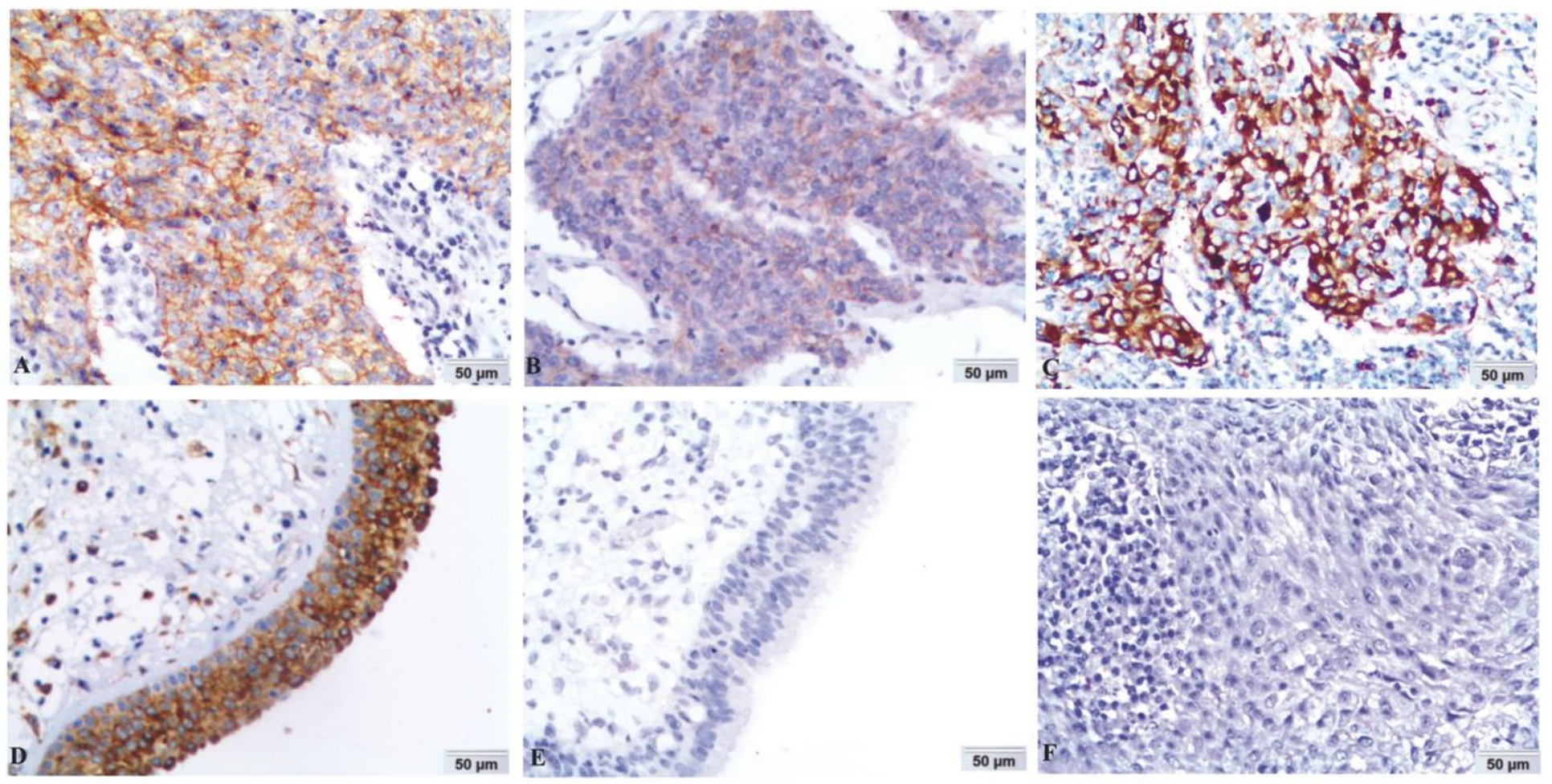

Figure 1

Expression of PD-L1, PD-1 and p-S6 proteins in NPC and non-cancerous nasopharyngeal epithelia was detected by immunohistochemistry (IHC). Positive expression of PD-L1 protein was located in the membrane of NPC (A). Positive expression of PD-1 protein was indicated in the cytoplasm of NPC (B). Strong staining of p-S6 protein was discovered in the cytoplasm of NPC (C). Positive staining of p-S6 protein was showed in the cytoplasm of control normal nasopharyngeal epithelia (D). No staining of PD-L1 protein was found in the control normal nasopharyngeal epithelia (E). Negative control was no staining in the NPC (F) (IHC, DAB staining, x200). 


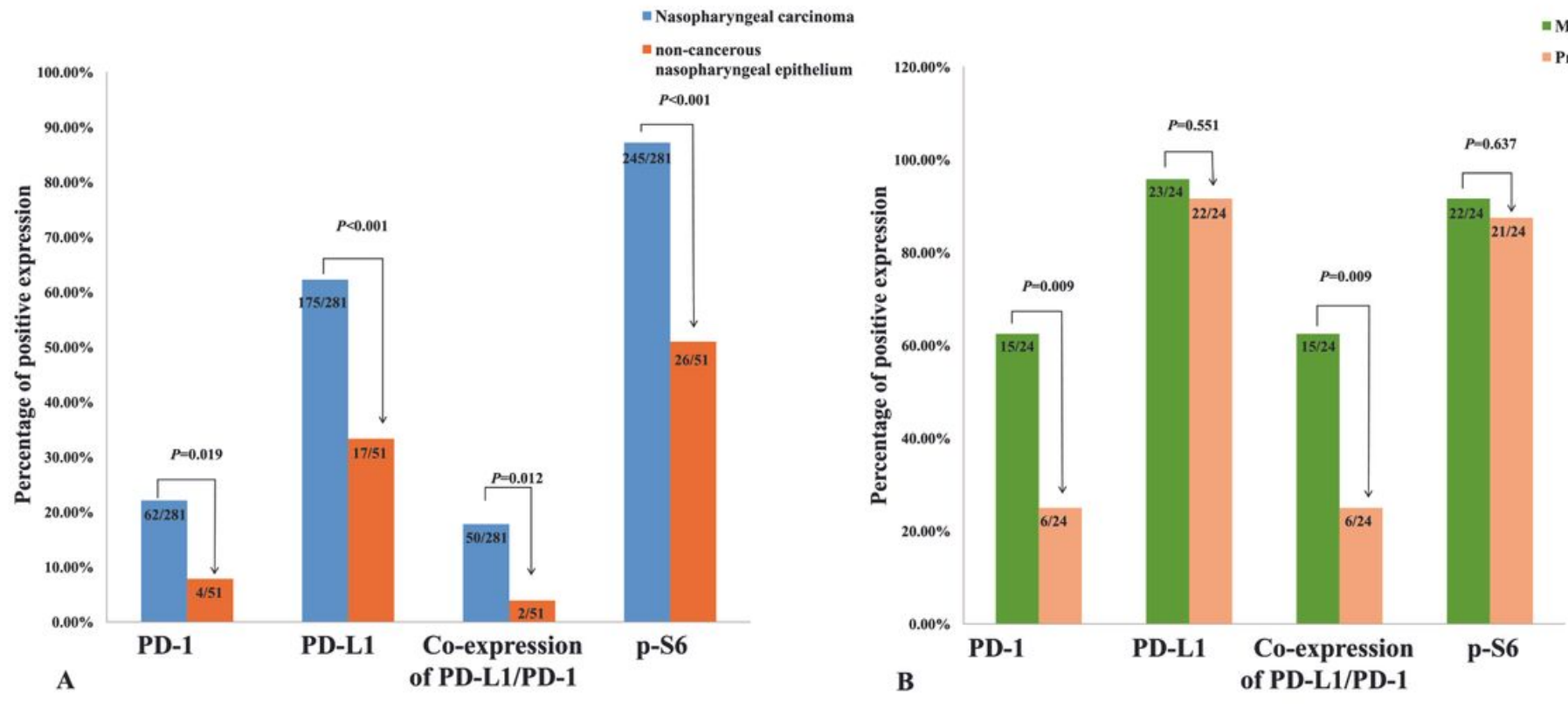

Figure 2

Positive expression of PD-1, PD-L1, p-S6 and common expression PD-1/PD-L1 in 281 cases of NPC and 51 cases of non-cancerous control nasopharyngeal epithelia, 24 pairs of primary NPC and their corresponding metastases were analyzed by $\chi 2$ test. A, The percentage of positive expression of PD-1, PD-L1, p-S6 and common expression PD-1/PD-L1 in NPC were evidently higher than those in the non-cancerous nasopharyngeal epithelia (all P<0.05). B, There were significantly higher expression of PD-1 and co-expression of PD-1/PD-L1 in metastatic NPC compared to the matched primary NPC (all P<0.01).
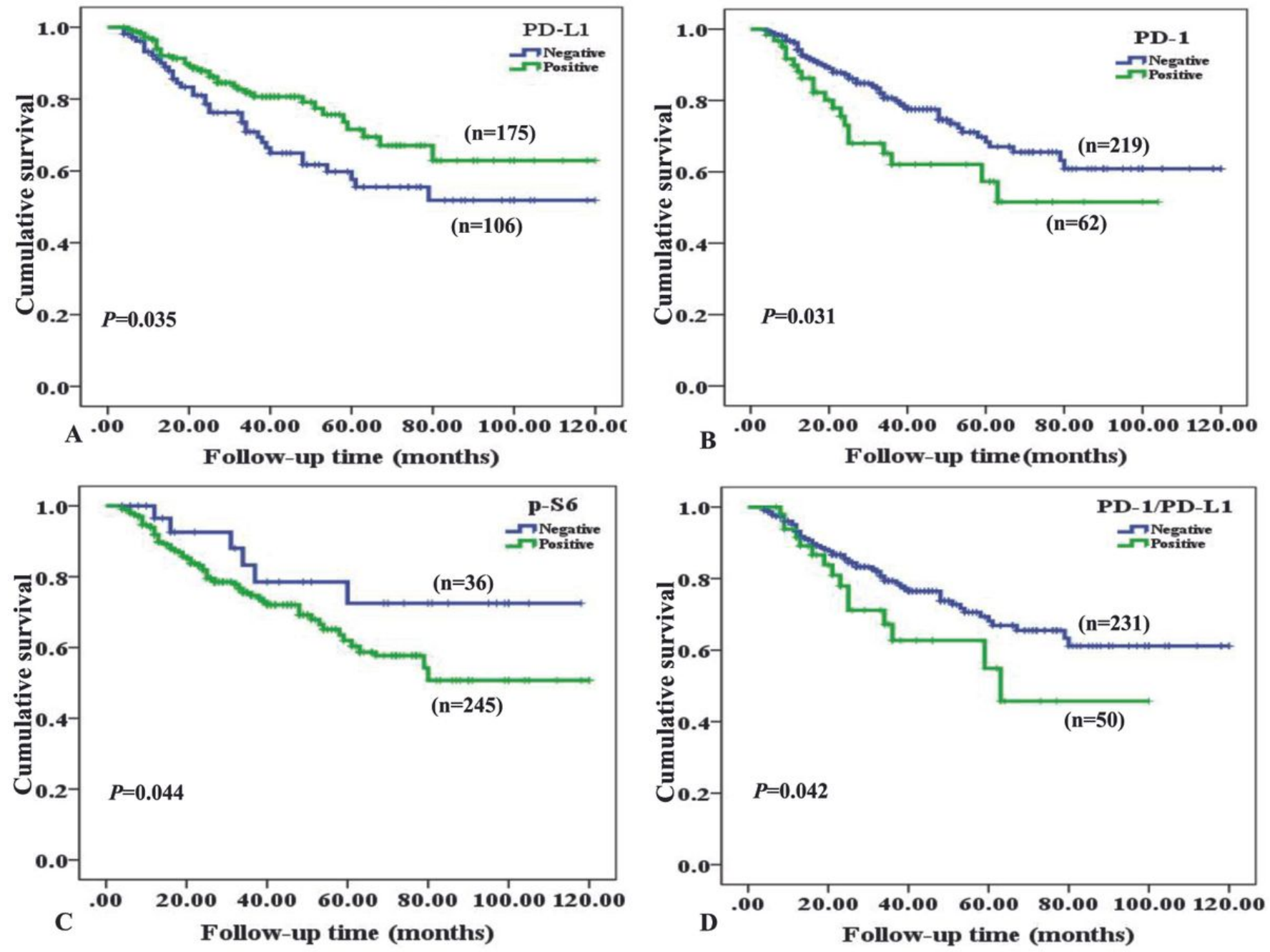
Figure 3

Kaplan-Meier curves for overall survival of NPC patients with expression of PD-L1, PD-1, p-S6 and co-expression of PD-L1/PD-1 assessed using the log-rank test (all tests were 2-sided). A『NPC patients with positive PD-L1 expression had a longer OS (overall survival) $(P=0.035)$; $B$ : NPC patients with negative PD-1 had a longer OS ( $P=0.031)$; : NPC patients with negative $p-S 6$ had a longer OS $(P=0.044)$; : NPC patients with common negative PD-L1/PD-1 had a longer OS $(P=0.042)$.

\section{Supplementary Files}

This is a list of supplementary files associated with this preprint. Click to download.

- TableS1.docx 